\title{
Effect of XingPiJieYu decoction on spatial learning and memory and CAMP-PKA-CREB- BDNF pathway in rat model of depression through chronic unpredictable stress
}

Chunye Wang ${ }^{1}$, Jianyou Guo ${ }^{2^{*}}$ and Rongjuan Guo ${ }^{1 *}$

\begin{abstract}
Background: Depression is a mental disorder characterized by a pervasive low mood and loss of pleasure or interest in usual activities, and often results in cognitive dysfunction. The disturbance of cognitive processes associated with depression, especially the impairment of learning and memory, exacerbates illness and increases recurrence of depression. XingPiJieYu (XPJY) is one of the most widely clinical formulas of traditional Chinese medicine (TCM) and can improve the symptoms of depression, including learning and memory. However, its regulatory effects haven't been comprehensively studied so far. Recently, some animal tests have indicated that the cyclic adenosine monophosphate (CAMP)-protein kinase A (PKA)-cAMP response element-binding protein (CREB)brain derived neurotrophic factor (BDNF) signaling pathway in hippocampus is closely related to depression and the pathogenesis of cognitive function impairments. The present study was performed to investigate the effect and mechanism of XPJY on depression and learning and memory in animal model.
\end{abstract}

Materials: The rat model of depression was established by chronic unpredictable stress (CUS) for 21 days. The rats were randomly divided into six groups: control group, CUS group, CUS + XPJY $(1.4 \mathrm{~g} / \mathrm{kg}, 0.7 \mathrm{~g} / \mathrm{kg}$ and $0.35 \mathrm{~g} / \mathrm{kg})$ groups, and CUS + sertraline (10 mg/kg) group. The sucrose preference, open field exploration and Morris water maze (MWM) were tested. The expression of CAMP, CREB, PKA and BDNF protein in hippocampus was examined with Elisa and Western Blot. The mRNA level of CREB and BDNF in hippocampus was measured with PCR.

Results: The results demonstrated that rats subjected to CUS exhibited decreases in sucrose preference, total ambulation, percentage of central ambulation, rearing in the open field test and spatial performance in the MWM. CUS reduced the expression of CAMP, PKA, CREB and BDNF in hippocampus of model rats. These effects could be reversed by XPJY.

Conclusion: The results indicated that XPJY can improve depression and related learning and memory and the effect of XPJY is partly exerted through the CAMP-PKA-CREB-BDNF signaling pathway.

Keywords: Chinese herbs, Depression, Learning and memory, Chronic unpredictable stress, CAMP, PKA, CREB, BDNF

\footnotetext{
* Correspondence: guojy@psych.ac.cn; dfguorongjuan@163.com

${ }^{2}$ CAS, Key Laboratory of Mental Health, Institute of Psychology, Chinese

Academy of Sciences, 100101 Beijing, China

${ }^{1}$ Dongfang Hospital Beijing University of Chinese Medicine, 100078 Beijing,

China
} 


\section{Background}

Depression is a mental disorder characterized by a pervasive low mood and loss of pleasure or interest in usual activities [1] and is the most common psychiatric illness that involves the disturbance of mood, with 10 to $20 \%$ lifetime prevalence [2]. The patients also demonstrate sleeplessness and suicidal tendencies, decreased foodintake and body-weight $[3,4]$, and more importantly always suffer from obvious cognitive function impairments, such as delayed thinking correlation, reduced computational capability, learning and memory impairment, and reduction in attention, comprehension and judgment [5-7]. Learning and memory impairment is one of the important residual symptoms, which has a strong impact on function of patients both at home and workplace. On the other hand, it is becoming increasingly clear that the disturbance of cognitive processes, especially the impairment of learning and memory, plays an important role in the development and complete recurrence of depression $[8,9]$. First of all, late-life depression is a risk factor for cognitive decline [10]. Furthermore, cognitive impairment is one of the typical features of recurrent depressive disorder (rDD), predominantly connected with episodic memory processes and the frontal functions (working memory) [11-13]. Cognitive impairment, linked with the earlier onset of depressive symptoms and episode prolongation, may in return lead to an ineffective antidepressant therapy and impede full recovery [14]. So it is the demand of reality and also an important task not to be avoided by mechanism research.

At present, depression is commonly treated with monoamine-based antidepressants such as selective serotonin reuptake inhibitors (SSRI). However, about $1 / 3$ of depressive patients are not responsive to conventional antidepressants [15], and these drugs is associated with a delay in symptom remission [16], severe side effects [17] and unsatisfactory outcome in cognition. Therefore, the treatment of depression is still a big problem and requires more effective approaches.

Traditional Chinese Medicine (TCM) has a long history in preventing and treating depression in Asia and is receiving more and more attention [18-22]. XPJY is a common prescription in clinical practices and shows relatively satisfactory therapeutic effect in improving depression and learning and memory level. Previous studies have shown that XPJY could improve behaviour and antidepression in chronic unpredictable stress model of depression in rats, which was as good as sertraline, and it also could reverse serum 5-HT and corticosterone from depression rats. Another study found that XPJY has the better effect on improving learning and memory ability in depression rats than sertraline, which might he related to reduce the inflammatory factors level, such as
IL-1 $\beta$, IL- 6 and TNF- $\alpha$, in serum and hippocampus. Recently, some animal tests have indicated that the cyclic adenosine monophosphate (cAMP)-protein kinase A (PKA)-cAMP response element-binding protein (CREB)brain derived neurotrophic factor (BDNF) signaling pathway in hippocampus is closely related to depression and the pathogenesis of cognitive function impairments [23-26]. However, the molecular mechanisms remain unclear and require verification in animals. A further study should be done this time. XPJY was composed of the following dried raw materials: acori tatarinowii rhizoma (Shi-Chang-pu), American ginseng (Xi-Yang-shen), radix curcumae (Yu-Jin) and prepared rehmannia root (Shoo-Di-huang). All of these were produced in accordance with the China Pharmacopoeia standard of quality control.

In the present study, we investigated the effects of XPJY on rats with depression established by chronic unpredictable stress and on the expression of cAMP-PKACREB-BDNF signal pathway.

\section{Methods}

Preparation and compositional analysis of XPJY

XPJY was composed of the following granule, which were derived from dried raw materials, including acori tatarinowii rhizoma (Shi-Chang-pu), American ginseng (Xi-Yang-shen), radix curcumae (Yu-Jin) and prepared rehmannia root (Shoo-Di-huang), in weight ratio of 0.5:1.2:2:3. All granules were bought from medicinal Materials Company of Beijing KangRenTang Company (Beijing, China), and analyzed for composition by high performance liquid chromatography (HPLC). The test solution was prepared by dissolving XPJY in methanol, and analyzed on an Agilent 1200 HPLC system with an C18 analytical column $(250 \times 4.6 \mathrm{~mm}, 5 \mu \mathrm{m})$. The mobile phase was consisted of water and acetonitrile in gradient elution. For quality control, the ginsenosides Rg1, $\mathrm{Re}, \mathrm{Rb} 1$ and acteoside were used as standard constituents. All the standards were purchased from National Institutes for Food and Drug Control (Beijing, China). As shown in Fig. 1, four bioactive compounds including

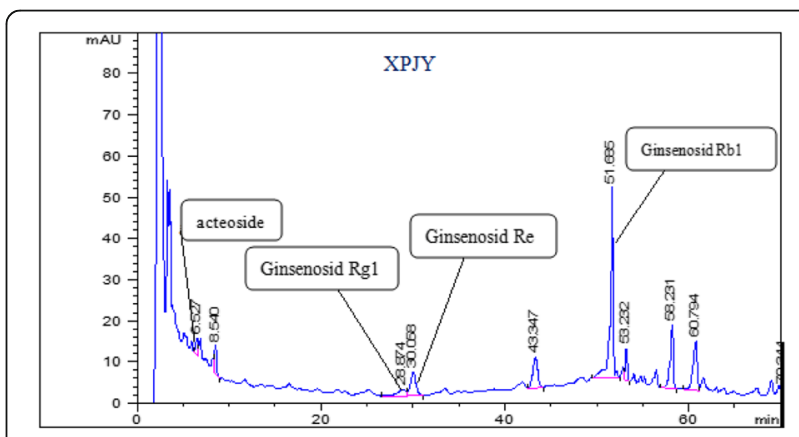

Fig 1 The HPLC Chromatogram of XPJY 
ginsenosides Rg1, Re, Rb1 and acteoside were found and exhibited high stability in XPJY by HPLC.

\section{Animals and grouping}

Adult male Sprague-Dawley rats (200-220 g) provided by Beijing Academy of Military Medical Sciences Laboratory Animal Centre were individually kept on a 12:12 $\mathrm{h}$ light/dark cycle in home cages with food and water available ad libitum except as described in stress. The experiment was approved by the Animal Ethic Committee of the university. Sixty rats (10 rats in each group) were randomly divided into six groups: control group receiving once-daily oral gavage (PO.) administration of distilled water for 21 days, CUS group receiving CUS and once-daily PO. administration of distilled water for 21 days, CUS + sertraline group receiving CUS and once-daily Po. sertraline at $10 \mathrm{mg} / \mathrm{kg}$ (Pfizer Inc., USA) for 21 days, CUS and XPJY groups receiving CUS and once-daily PO. XPJY at $0.35,0.7$ and $1.4 \mathrm{~g} / \mathrm{kg}$, respectively, for 21 days. The sertraline diluted in distilled water and XPJY were orally given $1 \mathrm{~h}$ before CUS exposure. The dosage " $0.7 \mathrm{~g} / \mathrm{kg}$ " has been calculated based mainly on the dosages of humans and animals. Then the double dosage was considered as one group, half dosage as another, hereby it works out that dose of XPJY is made as three groups: $0.35,0.7$ and $1.4 \mathrm{~g} / \mathrm{kg}$.

\section{Animal model preparation}

The CUS procedure was modified from the procedures used by Heine et al. [27]. Briefly, rats were exposed to different stressors daily for 21 days as follows: day1, cold immobilization for $1 \mathrm{~h}$ at $4{ }^{\circ} \mathrm{C}$, forced swim for $30 \mathrm{~min}$ at $25{ }^{\circ} \mathrm{C}$; day 2 , immobilization for $1 \mathrm{~h}$, crowding for $23 \mathrm{~h}$; day 3 , forced cold swim stress for $5 \mathrm{~min}$ at $10{ }^{\circ} \mathrm{C}$, isolation for $23 \mathrm{~h}$; day 4, immobilization for $1 \mathrm{~h}$, vibration for $1 \mathrm{~h}$; day 5 , forced swim stress for $30 \mathrm{~min}$ at $25^{\circ}$ $\mathrm{C}$, cold immobilization for $1 \mathrm{~h}$ at $4{ }^{\circ} \mathrm{C}$; day 6 , forced cold swim stress for $5 \mathrm{~min}$ at $10^{\circ} \mathrm{C}$, crowding for $23 \mathrm{~h}$; day 7 , vibration for $1 \mathrm{~h}$, isolation for $23 \mathrm{~h}$. This schedule was repeated twice for a total of 21 days. Prior to the study, certain criteria were set for excluding animal on weight loss, or the possible occurrence of wounds. Rats were acclimated to $3 \mathrm{~min}$ of handling once a day for 7 consecutive days before being used in experiment and were weighed on the 1st and 21th day of handling.

\section{Sucrose preference test}

Sucrose preference test was used to define anhedonia as a reduction in sucrose intake and sucrose preference. The sucrose preference test consisted of firstly removal of the food and water from the cage for a period of $20 \mathrm{~h}$. Then water and $1 \%$ sucrose were placed back to the cage and animals were allowed to consume the fluids freely for a period of $1 \mathrm{~h}$. The preference test was performed twice, separated by at least 5 days to calculate the mean for baseline. Then the preference test was conducted following the 21 days CUS period. On the last stress day, rats were deprived of water and food for $20 \mathrm{~h}$ and from the next day were given $1 \mathrm{~h}$ sucrose preference test (24 h after the last drug treatment). Sucrose and water consumption $(\mathrm{ml})$ was measured and the sucrose preference was calculated as the sucrose preference $(\%)=$ sucrose consumption/(sucrose consumption + water consumption).

\section{Open field exploratory behavior test}

Open field test was used to study the exploratory and anxiety behaviors of rats and was performed after the sucrose preference test. The rat was placed in the central square and observed for 5 min by a video camera and taped for further analysis. Motility was scored when the rat crossed a sector border with both hind-limbs. The following behaviors were scored by an observer who was blind to the drug treatment: central ambulation, number of central squares crossed; total ambulation, the overall number of peripheral and central square crossed; rearing, number of times the animal stood on its hind limbs; grooming, number of times the animal made these responses viz. grooming of the face, licking/cleaning and scratching the various parts of the body; immobility period, the time spent immobile. Anxiety-related behavior was measured by the percentage of central ambulation and calculated as the percentage of central ambulation $(\%)=$ central ambulation/total ambulation. Between tests, the apparatus was cleaned with $5 \%$ alcohol.

\section{Morris water maze test}

After the final stress stimulation, the Morris water maze (MWM) was used to determine the spatial learning and memory of the rats. The behavior of the animal was monitored with a video camera mounted in the ceiling above the centre of the pool. For oriented navigation test, the rats were trained for $120 \mathrm{~s}$ per trial and 4 trials per day starting at four different positions with $30 \mathrm{~min}$ intervals for 4 consecutive days as acquisition trials. Each trial began with the rat in the pool facing the sidewalls. When the rat escaped onto the platform, the rat was allowed to stay on the platform for $30 \mathrm{~s}$ before being returned to home cage. If the rat failed to escape within $120 \mathrm{~s}$, it was guided to the platform by the experimenter and allowed to stay for $15 \mathrm{~s}$. Each rat was trained once in the morning and once in the afternoon, and the time it took to find the platform (the latency period) was recorded. If the rats could not find the platform within $120 \mathrm{~s}$, they were taken out the water and the time was recorded as $120 \mathrm{~s}$. For the spatial exploration test, the hidden platform was removed on day 5 , and memory retrieval was examined by a probe trial that lasted for $120 \mathrm{~s}$. The escape latency in the acquisition trials, the 
times of crossing the platform position and the time spent in the target quadrant in the probe test were recorded by a computerised video tracking system.

\section{Measurement of the expression level of cAMP level}

After the last behavior test, hippocampus was taken out and the cAMP content was evaluated using EliteTM cAMP ELISA Assay Kit (eEnzyme, Montgomery Village, MD, USA). Extracted supernatants in each sample were loaded into EliteTM cAMP ELISA Assay Kit plates and each procedure was performed according to the manufacturer's instructions. After color development, the absorbance was measured at $\mathrm{Ex} / \mathrm{Em}=540 / 590 \mathrm{~nm}$ with fluorescence reader (Bio-Rad, CA, USA).

\section{Measurement of PKA, CREB, BDNF protein expressions by western bolt}

After the last behavior test, rats were sacrificed. Then the hippocampus was dissected, put into chilled tubes treated with an enzyme inhibitor and homogenized for Western blot analysis as previously reported [27], using primary antibodies for PKA, CREB and BDNF (1:2000, Santa Cruz Biotechnology) and $\beta$-actin (1:10000, Santa Cruz Biotechnology) followed by secondary antibodies conjugated with horseradish peroxidase (HRP, 1:5000, Bio-Rad). Immunoblots were visualized on X-ray film by chemiluminescence reaction (Pierce), and image analysis was performed on optical density calibrated images by AlphaEase Stand Alone software (Alpha Innotech). All experiments were performed 3 times.

\section{Evaluation of mRNA expression levels of CREB and BDNF by polymerase chain reaction (PCR)}

Total RNA from the hippocampus was isolated with RNASure $^{\circ}$ Mini Kit according to the manufacturer's protocol. The concentration of extracted RNA was calculated from the absorbance at $260 \mathrm{~nm}$ and the quality of RNA was assessed by absorbance at 260 and $280 \mathrm{~nm}$, with an acceptable ratio of A260 to A280 ranging from 1.9 to 2.1. Total RNA (1.5 mg) was transcribed using a high capacity cDNA reverse transcription kit (The Maxima ${ }^{\bullet}$ First Strand cDNA Synthesis Kit) according to the manufacturer's protocol. Real-time quantitative polymerase chain reaction (qPCR) analysis was performed with a Maxima ${ }^{\circ}$ SYBR Green qPCR Master Mix (2X), using the StepOnePlusTM real-time PCR system (Applied Bio systems, Inc., Foster City, CA). The details of all oligonucleotide primer sequences are listed in Table 1 . The PCR reaction system was denaturation at $95^{\circ}$ $\mathrm{C}$ for $15 \mathrm{~min}$ followed by 40 cycles of $94{ }^{\circ} \mathrm{C}$ for $15 \mathrm{~s}, 55^{\circ} \mathrm{C}$ for $30 \mathrm{~s}$ and $72{ }^{\circ} \mathrm{C} 45 \mathrm{~s}$, with final extension at $72{ }^{\circ} \mathrm{C}$ for $10 \mathrm{~min}$. Sequence Detection System Software (Version 1.0, Applied Bio systems, Inc., Foster City, CA) was used for data analysis. The relative expression of BDNF and CREB mRNAs was normalized to the amount of GAPDH.

\section{Statistical analysis}

The data were expressed as mean \pm S.E.M and analyzed by one-way ANOVA followed by Dunnet's multiple comparisons using GraphPad Prism 4.0. $p<0.05$ was set as significant level.

\section{Results \\ Body weight measurement}

At the 1st day of the CUS period, rats from different groups showed no significant difference in body weight $(p>0.05)$. However, significant difference was observed among groups following 21 days of CUS as shown in Table $2(p<0.01)$. The CUS significantly attenuated the gain of body weight when compared to control group $(p<0.01)$. Treatment with XPJY at 0.7 and $1.4 \mathrm{~g} / \mathrm{kg}$ significantly prevented the inhibition of body weight gain induced by CUS $(p<0.01)$; however, $0.35 \mathrm{~g} / \mathrm{kg}$ XPJY had no effect on the body weight gain induced by CUS $(p>0.05)$. In addition, $10 \mathrm{mg} / \mathrm{kg}$ sertraline also prevented the effect of CUS on body weight gain at day 21 of treatment $(p<0.01$; Table 2).

\section{Sucrose preference tests}

Before the CUS period, rats from different groups showed no significant difference in sucrose preference at day 1 . The sucrose preference was significantly reduced in the CUS group relative to the control group after exposure to CUS for 21 days $(p<0.01)$. Chronic treatment with XPJY at $1.4 \mathrm{~g} / \mathrm{kg}$ and sertraline at $10 \mathrm{mg} / \mathrm{kg}$ significantly suppressed the CUS-induced decrease in sucrose preference $(p<0.05)$. However, XPJY at $0.7 \mathrm{~g} / \mathrm{kg}$ and $0.35 \mathrm{~g} / \mathrm{kg}$ had no effect on the effect in sucrose preference induced by CUS ( $p>0.05$; Table 2$)$.

\section{Open field exploratory behavior test}

The result indicated CUS rats exhibited decreased total ambulation, percentage of center ambulation and rearing in comparison to control rats $(p<0.01$ or $p<0.05)$. XPJY $1.4 \mathrm{~g} / \mathrm{kg}$ and sertraline $10 \mathrm{mg} / \mathrm{kg}$ significantly reversed the CUS-induced behavioral alterations, as observed by increased total ambulation, percentage of central ambulation and rearing as compared to the CUS group $(p<0.05)$. XPJY $0.7 \mathrm{~g} / \mathrm{kg}$ and $0.35 \mathrm{~g} / \mathrm{kg}$ treatment didn't affect the stress induced behavior alterations in open field test (Table 3).

\section{Oriented navigation and spatial exploration tests}

The MWM test indicated that the escape latency in the CUS group was significantly prolonged compared to the control group on day 1 and $2(p<0.01)$. In comparison to the CUS group, treatment with XPJY $1.4 \mathrm{~g} / \mathrm{kg}$ significantly shortened the escape latency on day $1(p<0.05)$, while the treatments with XPJY at 0.35 and $0.7 \mathrm{~g} / \mathrm{kg}$ and sertraline at $10 \mathrm{mg} / \mathrm{kg}$ did not show significant effect on 
Table 1 Sequences of PCR primers

\begin{tabular}{|c|c|c|c|c|}
\hline Gene & Primes & Nucleotide sequences5'-3' & Product size (bp) & Accession No. \\
\hline$\overline{\mathrm{BDNF}}$ & Forward Reverse & $\begin{array}{l}\text { 5'-TGTGCGACAGCATTAGTGAG-3' } \\
\text { 5'-GCGTAGTTCGGCATTGGGAG-3' }\end{array}$ & 216 & XM_004418547 \\
\hline CREB & $\begin{array}{l}\text { Forward } \\
\text { Reverse }\end{array}$ & $\begin{array}{l}\text { 5'-TGAGTTGGCAAGTCCATTCG -3' } \\
\text { 5'- AACGGGCTATCCTGGTGAGT -3' }\end{array}$ & 156 & NM_012922 \\
\hline GAPDH & $\begin{array}{l}\text { Forward } \\
\text { Reverse }\end{array}$ & $\begin{array}{l}\text { 5'- CGGCAAGTTCAACGGCACAG -3' } \\
\text { 5'- CGCCAGTAGACTCCACGACAT -3' }\end{array}$ & 143 & NM_017008 \\
\hline
\end{tabular}

the escape latency induced by CUS ( $p>0.05$; Table 4$)$. In spatial test, CUS impaired memory retrieval as indicated by fewer crossing times of the platform position, while sertraline $10 \mathrm{mg} / \mathrm{kg}$ and XPJY $1.4 \mathrm{~g} / \mathrm{Kg}$ treatments restored the CUS-induced impairment of memory retrieval to levels seen in controls. XPJY 0.35 and $0.7 \mathrm{mg} / \mathrm{kg}$ had no effect on the memory retrieval impairment by CUS $(p>0.05$; Table 4$)$.

\section{Effects of XPJY on the expression of CAMP}

In comparison to the control group, the expression of protein cAMP significantly decreased in the hippocampus of the rats in CUS group $(p<0.01)$. The treatments with XPJY $1.4 \mathrm{~g} / \mathrm{kg}$ and sertraline $10 \mathrm{mg} / \mathrm{kg}$ significantly increased the expression of protein cAMP when compared to CUS group $(p<0.01$ or $p<0.05)$. However, XPJY at 0.35 and $0.7 \mathrm{~g} / \mathrm{kg}$ had no significant influence on the decrease of cAMP induced by CUS ( $p>0.05$; Fig. 2$)$.

\section{Effects of XPJY on the expression of PKA, CREB and BDNF proteins}

In comparison to the control group, the expression of protein PKA, CREB and BDNF significantly decreased in the hippocampus of the rats in CUS group $(p<0.01)$. The treatments with XPJY $1.4 \mathrm{~g} / \mathrm{kg}$ and sertraline $10 \mathrm{mg} / \mathrm{kg}$ significantly increased the expression of protein PKA, CREB and BDNF when compared to CUS group $(p<0.01)$. The treatments with XPJY $0.7 \mathrm{~g} / \mathrm{kg}$ significantly increased the expression of protein CREB when compared to CUS group $(p<0.05)$ However, XPJY at 0.35 and $0.7 \mathrm{~g} / \mathrm{kg}$ had no significant influence on the decrease of PKA, BDNF induced by CUS $(p>0.05)$. $\mathrm{XPJY}$ at 0.35 had no significant influence on the decrease of CREB induced by CUS ( $p>0.05$; Fig. 3 ).

\section{Expression of CREB and BDNF mRNAs}

The result indicated that the expression of CREB and BDNF mRNAs was significantly decreased by CUS when compared to control group $(p<0.01$ or $p<0.05)$. Chronic treatments with XPJY prevented the reduction of CREB and BDNF mRNA expression by CUS in a dosedependent manner. Treatment with XPJY $1.4 \mathrm{~g} / \mathrm{kg}$ and $0.7 \mathrm{~g} / \mathrm{kg}$ significantly prevented the CUS-induced reduction of CREB and BDNF mRNAs expression $(p<0.01$ or $p<0.05)$, however XPJY $0.35 \mathrm{~g} / \mathrm{kg}$ had no effect $(p>0.05)$. Similarly, sertraline $10 \mathrm{mg} / \mathrm{kg}$ treatment also markedly increased the expression of BDNF mRNAs ( $p<0.01$; Fig. 4$)$.

\section{Discussion}

The present study utilized broadly accepted traditional animal models - CUS model to examine depressive behaviors, the spatial learning and memory capability and the expressions of mRNAs/proteins of the cAMP-PKACREB-BDNF signaling. The results indicated that the body weight gain, sucrose preference, total ambulation, percentage of central ambulation and rearing after CUS for 21 were significantly decreased. It indicates that the depression model was successfully established. Then the spatial cognitive performance in the Morris water maze task was also decreased, which demonstrated that depression by CUS had a dramatic influence on spatial cognitive performance in the MWN test, while the treatment of the rats with XPJY significantly reversed these changes. Furthermore, the expressions of mRNAs/proteins in the cascade of cAMP-PKA-CREB-BDNF signaling were decreased by CUS, too. The administration of $\mathrm{XPJY}$ to stressed rats prevented such metabolite reductions. These results suggest that XPJY could improve depression and related learning/memory impairment through the cAMP-PKA-CREB-BDNF signal cascade.

Table 2 The changes of animal body weight and sucrose preference in different groups

\begin{tabular}{|c|c|c|c|c|}
\hline \multirow[t]{2}{*}{ Groups } & \multicolumn{2}{|c|}{ Body weight (g) } & \multicolumn{2}{|c|}{ Sucrose preference (\%) } \\
\hline & 1st day & 21st day & 1st day & 21st day \\
\hline $\begin{array}{l}\text { Control } \\
\text { CUS } \\
\text { CUS + sertraline } 10 \mathrm{mg} / \mathrm{kg} \\
\text { CUS + XPJY } 1.4 \mathrm{~g} / \mathrm{kg} \\
\text { CUS + XPJY } 0.7 \mathrm{~g} / \mathrm{kg} \\
\text { CUS + XPJY } 0.35 \mathrm{~g} / \mathrm{kg}\end{array}$ & $\begin{array}{l}184.3 \pm 5.8 \\
183.4 \pm 9.0 \\
183.5 \pm 7.8 \\
180.6 \pm 10.0 \\
180.8 \pm 13.7 \\
181.9 \pm 13.2\end{array}$ & $\begin{array}{l}317.0 \pm 14.8 \\
244.0 \pm 38.8++ \\
301.5 \pm 19.2^{* *} \\
308.1 \pm 22.0^{* *} \\
294.5 \pm 16.6^{* *} \\
273.6 \pm 30.1\end{array}$ & $\begin{array}{l}0.865 \pm 0.082 \\
0.867 \pm 0.058 \\
0.857 \pm 0.064 \\
0.888 \pm 0.108 \\
0.878 \pm 0.102 \\
0.864 \pm 0.076\end{array}$ & $\begin{array}{l}0.874 \pm 0.091 \\
0.598 \pm 0.117++ \\
0.761 \pm 0.242^{*} \\
0.776 \pm 0.141^{*} \\
0.712 \pm 0.153 \\
0.682 \pm 0.186\end{array}$ \\
\hline
\end{tabular}

Note: $++p<0.01$ vs Control group; ${ }^{* *} p<0.01$ vs CUS group; ${ }^{*} p<0.05$ vs CUS group 
Table 3 The effects of XPJY on open field test in different groups

\begin{tabular}{llll}
\hline Groups & Total ambulation & Central ambulation (\%) & Rearing \\
\hline Control & $4749.1 \pm 835.3$ & $22.6 \pm 12.7$ & $9.1 \pm 3.2$ \\
CUS & $3834.2 \pm 588.1+$ & $6.3 \pm 4.3++$ & $3.4 \pm 2.1++$ \\
CUS + sertraline $10 \mathrm{mg} / \mathrm{kg}$ & $4616.4 \pm 499.6^{*}$ & $14.1 \pm 8.7^{*}$ & $7.8 \pm 3.5^{*}$ \\
CUS + XPJY $1.4 \mathrm{~g} / \mathrm{kg}$ & $4506.0 \pm 638.5^{*}$ & $16.2 \pm 8.1^{*}$ & $7.0 \pm 3.8^{*}$ \\
CUS + XPJY $0.7 \mathrm{~g} / \mathrm{kg}$ & $4335.8 \pm 773.1$ & $12.1 \pm 6.8$ & $5.4 \pm 3.8$ \\
CUS + XPJY $0.35 \mathrm{~g} / \mathrm{kg}$ & $4144.5 \pm 649.1$ & $9.7 \pm 12.8$ & $5.1 \pm 4.5$ \\
\hline
\end{tabular}

Note: $++p<0.01$ vs Control group; $+p<0.05$ vs Control group; ${ }^{*} p<0.05$ vs CUS group

Depression is a highly debilitating and widely distributed mental illness in the general population with a lifetime incidence of $15-25 \%$, ranking it as one of the most burdensome diseases of society [5-7], besides enormous personal suffering and increased mortality risk [28]. Depression may display many different syndromes like a pervasive low mood and loss of pleasure or interest in usual activities [1]. The patients also demonstrate sleeplessness, decreased body-weight [3, 4], and obvious cognitive function impairments, such as learning and memory impairment, etc. [5-7]. Cognitive function related to depression has received far more attention. A growing body of research suggests that depressive symptoms and cognitive impairment are common often coexist in an individual patient, especially in older adults. And late-life depression even is a risk factor for cognitive decline [10]. Another clinical research also found that depressed participants, in the normal aging older adults, had a lower performance compared to non-depressed participants in cognitive domains, and the depressive symptoms may have a distinct impact on cognitive performance [29]. The studies show that the pattern of cognitive impairment associated with depressive symptoms involves executive dysfunction, reduced processing speed, and deficits in episodic memory [30-32], while global intellectual ability, language skills, visuospatial abilities, and semantic processing are usually spared [33].

Among them, learning and memory impairment is one of the important cognitive impairments and residual symptoms, which has a strong impact on function of patients both at home and workplace, and the life quality of patients. Many studies also have reported learning and memory deficits in depressed subjects [3]. Further, it is becoming increasingly clear that the disturbance of cognitive processes, especially the impairment of learning and memory, plays an important role in the development and complete recurrence of depression [8, 9]. In addition, cognitive impairment is also one of the typical features of recurrent depressive disorder, predominantly connected with episodic memory processes and working memory [11-13]. And cognitive impairment, linked with the earlier onset of depressive symptoms and episode prolongation, may in return lead to an ineffective antidepressant therapy and impede full recovery [14].

In this study, the chronic unpredictable stress for 21 days was used to establish depression model of rat and simulate the long-term negative modes and life events of humans. The chronic unpredictable stress procedure is one of the well-validated animal models of depression [34], that has good face validity in rodents as it can elicit depression like symptoms such as lack of sucrose preference [35, 36] interpreted as anhedonia [37] and reduced locomotor activity [38]. Anhedonia-like behavior is the core symptom of human depression [39], which means inability to experience pleasure. Anhedonia has been defined as decreased responsiveness to rewards $[40,41]$, and it is measured originally by declining intake of a palatable sweet solution. In this experimental conditions, there has been a significantly reduction of sucrose preference in CUS group compared with the control, which was reduced to approximately $27 \%$ at day 21 after the beginning of stress exposure. Reduced locomotor activity of rats in open field test may mimic some aspects of human psychomotor retardation [42], which is an accompanying symptom of depression in humans [40]. In the experimental conditions, chronic unpredictable stress rats also exhibited depressive behavior which is displayed by decreased total ambulation, central ambulation, rearing in comparison to control rats. Depression may also display many other different syndromes like

Table 4 The effects of XPJY and sertraline on escapes latency in different groups

\begin{tabular}{llllll}
\hline \multirow{2}{*}{ Groups } & \multicolumn{2}{l}{ Escape latency $(\mathrm{s})$} & & \multirow{2}{*}{ Times crossing platform position } \\
\cline { 2 - 4 } & Day1 & Day2 & Day3 & Day4 & Day5 \\
\hline Control & $25.2 \pm 8.2$ & $23.3 \pm 6.7$ & $21.5 \pm 5.9$ & $18.1 \pm 4.1$ & $4.38 \pm 1.51$ \\
CUS & $38.8 \pm 7.6++$ & $32.9 \pm 9.2+$ & $23.5 \pm 5.1$ & $18.9 \pm 5.4$ & $2.13 \pm 1.13++$ \\
CUS + sertraline $10 \mathrm{mg} / \mathrm{kg}$ & $30.5 \pm 13.4$ & $27.7 \pm 7.3$ & $20.2 \pm 4.4$ & $17.6 \pm 4.3$ & $3.63 \pm 1.41^{*}$ \\
CUS + XPYY $1.4 \mathrm{~g} / \mathrm{kg}$ & $28.6 \pm 9.3^{*}$ & $25.3 \pm 9.6$ & $20.9 \pm 5.2$ & $17.5 \pm 7.3$ & $3.88 \pm 1.64^{*}$ \\
CUS + XPYY $0.7 \mathrm{~g} / \mathrm{kg}$ & $33.1 \pm 6.8$ & $27.7 \pm 10.7$ & $20.0 \pm 4.2$ & $17.8 \pm 5.4$ & $3.13 \pm 1.13$ \\
CUS + XPJY $0.35 \mathrm{~g} / \mathrm{kg}$ & $34.4 \pm 14.6$ & $28.7 \pm 9.8$ & $19.6 \pm 4.5$ & $18.9 \pm 5.0$ & $2.88 \pm 1.46$ \\
\hline
\end{tabular}

Note: $++p<0.01$ vs Control group, $+p<0.05$ vs Control group; $* p<0.05$ vs CUS group 


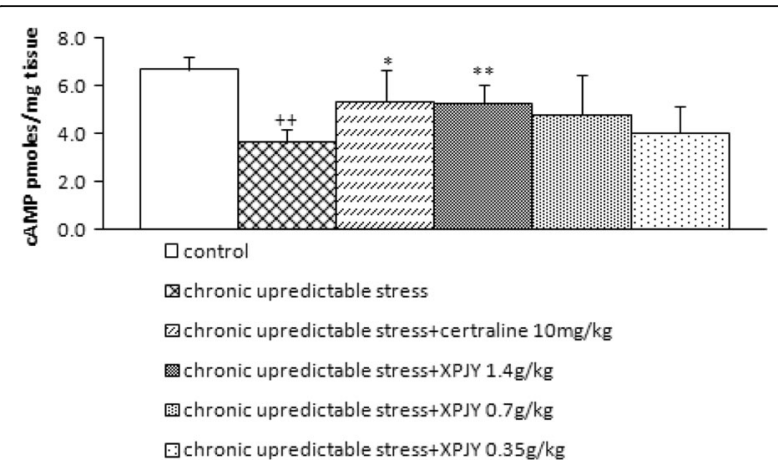

Fig. 2 Effect of XPJY on CAMP following 21 days of chronic unpredictable stress. CAMP was reduced in the chronic unpredictable stress group compared with control group $(P<0.01)$. Chronic treatment with XPJY $1.4 \mathrm{~g} / \mathrm{kg}$ significantly increase in CAMP compared with the CUS group $(P<0.01)$. Sertraline $10 \mathrm{mg} / \mathrm{kg}$ group also significantly increase in CAMP compared with the CUS group $(P<0.05)$. XPJY $0.7 \mathrm{~g} / \mathrm{kg}$ and $0.7 \mathrm{~g} / \mathrm{kg}$ treatment did not alter stressinduced CAMP alterations. $++P<0.01$, as compared to the control group; ${ }^{*} P<0.05$, as compared to the chronic unpredictable stress group

decreased body weight [43] and impaired learning/memory [44].

The Morris water maze has been widely used all over the world to detecting spatial learning memory capability [45], to objectively reflect their cognitive levels. The rats are trained to learn to use the relationship between environmental labels and latent platforms in order to judge the positions of the platforms in the water. They may thus form stable spatial cognition. The rats were allowed to swim from the original position to the latent platform under water by utilizing the indications at the distal end. Their spatial learning was evaluated by repeated training. When the platforms under water were withdrawn, the reference memory of the animals was determined by using the frequency of penetration of the platform position [7]. The present study found that the escape latency in the XPJY $1.4 \mathrm{~g} / \mathrm{kg}$ group on the first day was significantly shortened, but the difference for the sertraline group was not statistically significant. This results shows XPJY could increase the ability of spatial learning of depression rats by CUS. In contrast, the frequency for penetrating the central areas increased both in the XPJY $1.4 \mathrm{~g} / \mathrm{kg}$ group and in the sertraline group in the spatial exploration test on the fifth day. It indicated that they can both achieve improvement with regard to rats' memory capability, while the efficacy of the XPJY $1.4 \mathrm{~g} / \mathrm{kg}$ group was more significant. This results shows XPJY increased the ability of spatial learning memory better than sertraline.

Previous studies have shown that chronic stressful life events are major reactions for inducing depression and a decrease in learning memory capability [44], as the major target for stress. The experimental results are
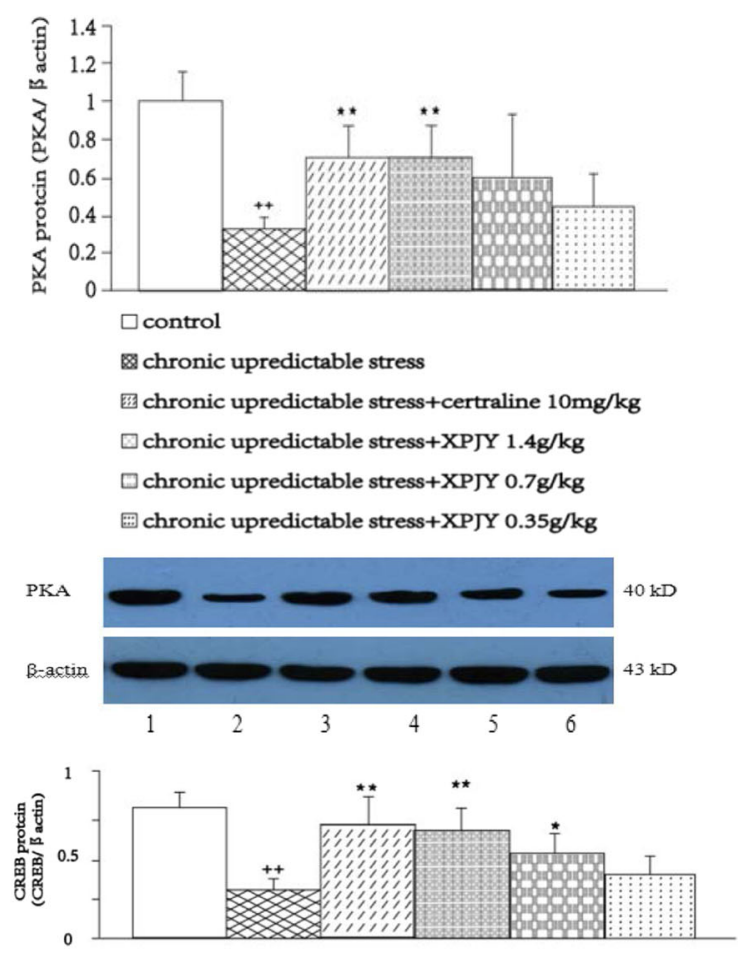

$\square$ control

\% chronic upredictable stress

国 chronic upredictable stress+certraline $10 \mathrm{mg} / \mathrm{kg}$

$\square$ chronic upredictable stress+XPJY $1.4 \mathrm{~g} / \mathrm{kg}$

$\square$ chronic upredictable stress+XPJY $0.7 \mathrm{~g} / \mathrm{kg}$

⿴囗十 chronic upredictable stress + XPIY $0.35 \mathrm{~g} / \mathrm{kg}$
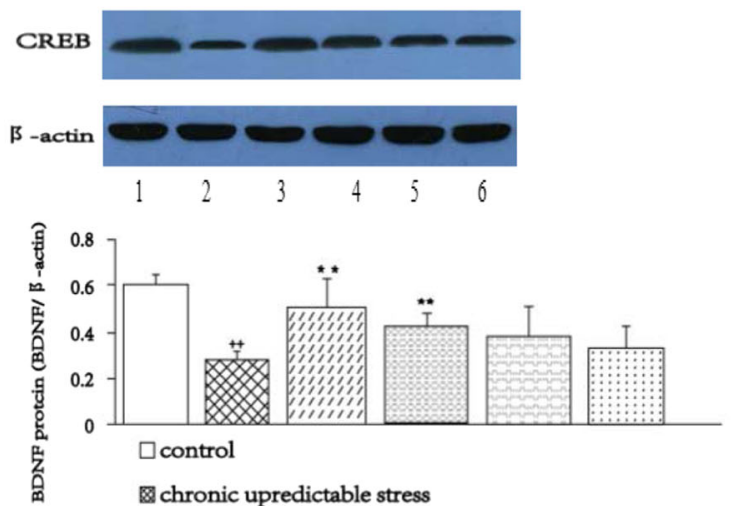

圈 chronic upredictable stress+certraline $10 \mathrm{mg} / \mathrm{kg}$

$\square$ chronic upredictable stress+XPJY $1.4 \mathrm{~g} / \mathrm{kg}$

$\square$ chronic upredictable stress+XPJY $0.7 \mathrm{~g} / \mathrm{kg}$

: $\mathrm{Phronic}$ upredictable stress+XPJY $0.35 \mathrm{~g} / \mathrm{kg}$

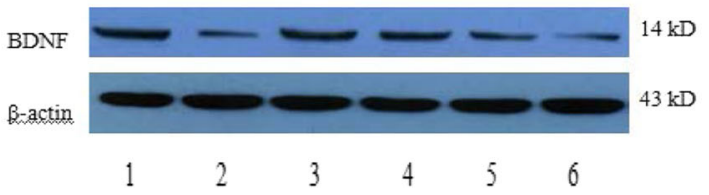


Fig. 3 Effect of XPJY on hippocampal PKA, CREB and BDNF protein level following 21 days of chronic unpredictable stress. Relative optical density (OD) of PKAVCREB/BDNF to $\beta$-actin. PKA, CREB and BDNF were reduced in the chronic unpredictable stress group compared with control group $(P<0.01)$. Chronic treatment with XPJY $1.4 \mathrm{~g} / \mathrm{kg}$ significantly increase in PKA, CREB and BDNF compared with the CUS group $(P<0.01)$. Sertraline $10 \mathrm{mg} / \mathrm{kg}$ group also significantly increase in PKA, CREB and BDNF compared with the CUS group $(P<0.01)$. XPJY $0.7 \mathrm{~g} / \mathrm{kg}$ group also significantly increase in PKA, CREB and BDNF compared with the CUS group $(P<0.05)$. XPJY $0.7 \mathrm{~g} / \mathrm{kg}$ and $0.35 \mathrm{~g} / \mathrm{kg}$ treatment did not alter stress-induced PKA, BDNF alterations $(P>0.05)$. $++P<0.01$, as compared to the control group; ${ }^{*} P<0.05$, as compared to the chronic unpredictable stress group. Representative immunoblots of PKA/CREB/BDNF and $\beta$-actin. 1, control; 2 , chronic unpredictable stress; 3, chronic unpredictable stress plus chronic sertraline (10 mg/kg); 4, chronic unpredictable stress plus chronic XPJY $(1.4 \mathrm{~g} / \mathrm{kg})$; 5, chronic unpredictable stress plus chronic XPJY (0.7 g/kg); 6, chronic unpredictable stress plus chronic XPJY $(0.35 \mathrm{~g} / \mathrm{kg})$

consistent with actual situation. The primary findings of the present study show that CUS causes cognitive decline and depression like symptoms whereas XPJY showed ameliorating potential against detrimental effect of CUS. These results are in agreement with several other studies which also showed that UCMS causes cognitive decline and depression like behavior in animal models $[38,46]$.

The neurobiological mechanisms connecting the depressive symptoms with cognitive and functional performance are heterogeneous and have not been completely elucidated. A number of studies have shown that abnormalities in the hippocampus are closely associated with the occurrence and development of depression [47-50]. Depression may affect learning memory capability by injuring hippocampus neurons [51], which has strong connections with depression and learning memory capability [52]. The mechanisms for cognitive disorder in depression mainly have two dimensions: neurobiological and vascular factors, which may mediate the cognitive and functional changes associated with depression [53], including changes in monoamine systems dysfunction, hormonal and immunologic changes, inflammatory processes, and alterations on genes expression [54]. Such as, the hypothalamic-pituitary-adrenal axis dysfunction in depression, which relates to hippocampal atrophy, may be a neurobiological causal factor to the episodic memory impairment in depressed subjects $[54,55]$; and white matter lesions as vascular burden have be found in depressed subjects [56]. Our earlier studies have shown that learning and memory ability in depression rats might be related to reduce the inflammatory factors level, such as IL-1 $\beta$, IL-6 and TNF$\alpha$, in serum and hippocampus [57].

Pathophysiological studies on depression have recently been gradually transferred to the intracellular secondary messenger system. As an intracellular secondary messenger,

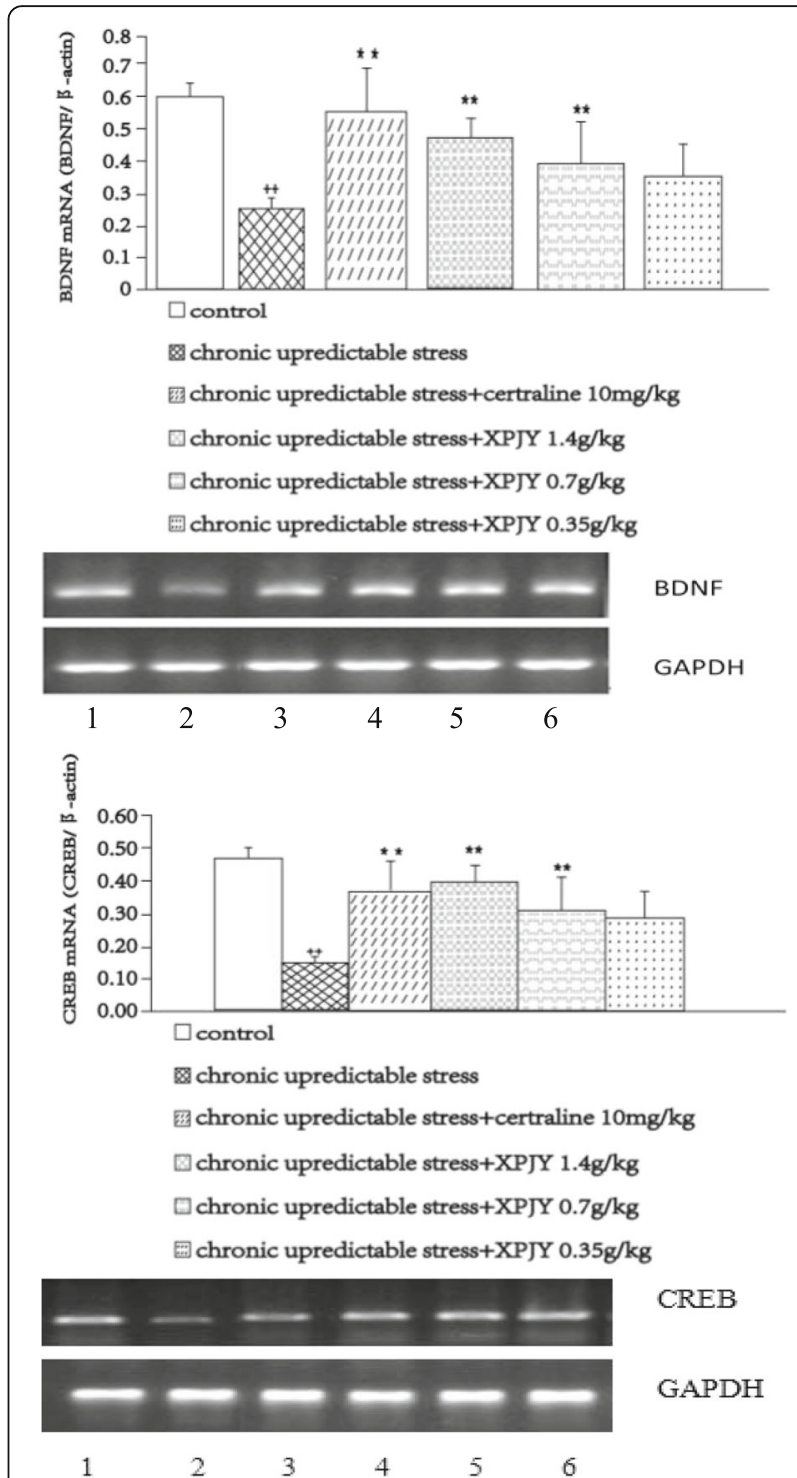

Fig. 4 CREB and BDNF mRNA expression following 21 days of chronic unpredictable stress. 1, control; 2, chronic unpredictable stress; 3, chronic unpredictable stress plus chronic XPJY $(0.35 \mathrm{~g} / \mathrm{kg}) ; 4$, chronic unpredictable stress plus chronic XPJY $(0.7 \mathrm{~g} / \mathrm{kg}) ; 5$, chronic unpredictable stress plus chronic XPJY (1.4 g/kg); 6, chronic unpredictable stress plus chronic sertraline $(10 \mathrm{mg} / \mathrm{kg})$. CREB and BDNF mRNA were reduced in the chronic unpredictable stress group compared with control group $(P<0.01)$. Chronic treatment with XPJY $1.4 \mathrm{~g} / \mathrm{kg}$ significantly increase in CREB and BDNF mRNA compared with the CUS group $(P<0.01)$. Sertraline $10 \mathrm{mg} / \mathrm{kg}$ group also significantly increase in CAMP compared with the CUS group $(P<0.01)$. Chronic treatment with XPJY $0.7 \mathrm{~g} / \mathrm{kg}$ significantly increase in CREB and BDNF mRNA compared with the CUS group $(P<0.01$ or $P<0.05)$. XPJY $0.35 \mathrm{~g} / \mathrm{kg}$ treatment did not alter stress-induced CREB and BDNF mRNA alterations. $++P<0.01$, as compared to the control group; ${ }^{*} P<0.05,{ }^{*} P<0.01$, as compared to the chronic unpredictable stress group 
cAMP can promote neuronal differentiation and survival $[58,59]$ as well as outgrowth, regeneration [60-62] and guidance of neuronal processes $[63,64]$, whose signaling has been shown to be implicated in mechanism of reduced synaptic plasticity, that may contribute to the pathophysiology of depression [65, 66]. cAMP can activate cAMPdependent protein kinase (PKA), and subsequently PKA is able to activate CREB directly by phosphorylation of the transcription factor CREB [67] or indirectly [58, 68], thus further mediating multiple signaling molecules, like CREB and BDNF which play important roles in the signaling pathway of learning memory and depression [10].

CREB is a kind of regulatory factor in nuclei, as an important component of multiple intracellular signaling pathways in the nervous system, and is capable of regulating transcription by autophosphorylation. Many intracellular signal transduction cascades can influence, directly or indirectly, the activation of CREB. Some of the enzymes that participate in these cascades are PKA, protein kinase $\mathrm{C}$ (PKC), Ca2+/calmodulindependent protein kinase (CAMKII), extracellular-regulated protein kinase (ERK), phosphoinositide 3-kinase (PI3K), and glycogen synthase kinase 3 (GSK-3) [69, 70]. The downstream actions of CREB include the influences on neuron synaptic plasticity and the formation of long term memory [71, 72]. CREB is critical for the formation of hippocampally-dependent long-term memory [73]. In addition, CREB also decreases in the brains of patients with depression, which are elevated in those patients who have been using antidepressants [74].

BDNF is one of the downstream target genes of CREB, which is the most prevalent neurotrophic factor in the brain. It can be induced by the phosphorylation of CREB and responsible for neuronal survival, maintenance and growth. Some studies show that a decreased expression of BDNF, a key target implicated both in the etiology of depression [75], appears to be associated with depression symptoms both in animals and humans. Depressive patients have a decrease in serum, plasma and hippocampal BDNF levels in depressive patients [76, 77]. And a decreased serum BDNF level may be an indicator of vulnerability to develop depression [78]. On the other hand, several animal models of depression have also shown a reduced expression of BDNF in brain regions [79-82] and produce an antidepressant-like behavior [83]. Furthermore, BDNF plays important roles in facilitating both early and late phase of LTP $[84,85]$.

Then, CREB can induce the expression of BDNF in general $[86,87]$, while BDNF can also activate the production of CREB [11]. They form a positive feedback ring. BDNF is the best studied neurotrophic factor implicated in depression, which is also concerned to neuroplasticity and memory. Previous studies have found that the expression level of BDNF in the hippocampus of
CUS induced mice markedly reduced [88]. BDNF also could adjust the plasticity of neurons, such as plasticity of 5-HT neurons in the central nervous system $[89,90]$.

It has also been indicated that the intracellular cAMPPKA-CREB-BDNF signaling pathway can be activated after anti-depression therapy for a period. [11]. Previous studies have revealed that the cAMP-PKA-CREB -BDNF signaling is involved in depressive behaviors in animals $[18,19,91]$. Consistently, the present study showed XPJY prevented the reduction of the CAMP-PKA-CREB -BDNF signaling cascade induced by CUS while improving the depressive behaviors. The most important, the present results show the CAMP-PKA-CREB -BDNF signaling may be also involved in the ability of spatial learning memory of depressive rats. The ability of spatial cognitive recovered with the increasement of CAMP-PKA-CREB -BDNF signaling, suggesting the effects of XPJY may be through regulation of the signaling pathway. Some recent studies also supported that the ability of learning and memory improved through cAMP-PKA-CREB-BDNF signaling pathway $[12,92]$. Interestingly, sertraline in the present study showed the effect of antidepression and increasement of signaling pathway as same as XPJY, but didn't in spatial learning memory test. The XPJY $1.4 \mathrm{~g} / \mathrm{kg}$ group was more advantageous than sertraline in improving the learning memory ethology of depression rats. There are two probabilities: sertraline may need more time to work, or XPJY also work though other signaling pathway about learning and memory. These studies indicated that XPJY plays antidepression and anti learning memory impairment effect through the cAMP-PKA-CREB -BDNF signaling pathway, which remains to be further studied using specific blockers of the signal pathway. Other mechanisms may also be involved, and this still requires further study.

Recently, TCM received more and more attention in treating depression and related syndromes [15-17]. $\mathrm{XPJY}$ is a common prescription in clinical practices and shows relatively satisfactory therapeutic effect in improving depression and cognitive dysfunction. In our previous studies, we established the depression model in the same way, and the expression of serum 5-HT and corticosterone were examined with Elisa. The results demonstrated that rats subjected to CUS exhibited decreases in serum 5-HT and increases in serum corticosterone. The results have shown that XPJY reversed the depression-like behaviors, increased serum 5-HT and decreased serum corticosterone induced by CUS in rats as the same as sertraline could [93]. In summary, the present study indicated that XPJY could improve the depression and related syndromes in rat CUS model through cAMP-PKA-CREB-BDNF pathway. These findings are important for understanding therapeutic effects of Chinese medicine XPJY on the impairment of learning and memory in depression. 


\section{Conclusions}

XPJY has regulatory effect on depressive behavior in the sucrose preference and open field exploration, and learning and memory in Morris water maze, and the expression of cAMP, PKA, CREB and BDNF in hippocampus of model rats. The effect is probably achieved mainly by activating cAMP-PKA-CREB-BDNF signaling pathway. This study provides experimental evidence for the clinical application of XPJY in the treatment of depression and related learning and memory impairment.

\section{Abbreviations \\ BDNF: Brain derived neurotrophic factor; CAMP: Cyclic adenosine monophosphate; CREB: CAMP response element-binding protein; CUS: Chronic unpredictable stress; MWM: Morris water maze; PKA: Protein kinase A; rDD: Recurrent depressive disorder; SSRI: Selective serotonin reuptake inhibitors; TCM: Traditional Chinese medicine; XPJY: XingPiJieYu decoction}

\section{Acknowledgements}

This research was supported by National Natural Science Foundation of China (No.81072717).

\section{Funding}

National Natural Science Foundation of China (No.81072717).

\section{Availability of data and materials}

We have presented all our main data in the form of figures and tables. The datasets supporting the conclusions of this article are included within the article.

\section{Authors' Contributions}

Chunye Wang carried out the PCR and Western blot studies and drafted the manuscript. Jianyou Guo participated in the design of the study and performed the statistical analysis. Rongjuan Guo conceived of the study, and participated in its design and coordination and helped to draft the manuscript. All authors read and approved the final manuscript.

\section{Competing interests}

The authors declare that they have no conflicts of interest to this work. We declare that we do not have any commercial or associative interest that represents a conflict of interest in connection with the work submitted.

\section{Consent for publication}

My article excludes human participants and clinical data.

\section{Ethics approval and consent to participate}

Procedures involving animals and their care were conducted in conformity with NIH guidelines (NIH Pub. No. 85-23, revised 1996) and was approved by Animal Care and Use Committee of the Beijing University of Chinese Medicine.

Received: 12 June 2016 Accepted: 22 December 2016

Published online: 24 January 2017

\section{References}

1. Chen PJ, Hsieh CL, Su KP, Hou YC, Chiang HM, Lin IH, Sheen LY. The antidepressant effect of Gastrodia elata Bl. on the forced-swimming test in rats. Am J Chin Med. 2008;36(1):95-106.

2. Wong ML, Licinio J. Research and treatment approaches to depression. Nat Rev Neurosci. 2001;2(5):343-51.

3. Zakzanis KK, Leach L, Kaplan E. On the nature and pattern of neurocognitive function in major depressive disorder. Neuropsychiatry Neuropsychol Behav Neurol. 1998;11(11):111-9.

4. Banerjee R, Ghosh KA, et al. Stress. Negative Modulator of NGF. 2011;1(2):1-7.

5. Kessler RC, Berglund P, Demler O, et al. National Comrobidity Survey Replication. The epidemiology of major depressive disorder: results from the National Comorbidity Survey Replication (NCS-R). JAMA. 2003;289(23):3095-105.
6. Nemeroff CB. The burden of severe depression: a review of diagnositic challenges and treatment alternatives. J Psychiatr Res. 2007;41(3-4):189-206.

7. Patten SB. Major depression prevalence is very high, but the syndrome is a poor proxy for community populations' clinical treatment needs. Can J Psychiatry. 2008;53(7):411-9.

8. Dolan RJ. Emotion, cognition, and behavior. Science. 2002;298(5596):1191-4

9. Sun MK, Alkon DL. Induced depressive behavior impairs learning and memory in rats. Neuroscience. 2004;129(1):129-39.

10. Diniz BS, Butters MA, Albert SM, Dew MA, Reynolds CFIII. Late-life depression and risk of vascular dementia and Alzheimer's disease: systematic review and meta-analysis of community-based cohort studies. Br J Psychiatry. 2013; 202(5):329-35.

11. Lee RSC, Hermens DF, Porter MA, Redoblado-Hodge MA. A meta-analysis of cognitive deficits in first-episode Major Depressive Disorder. J Affect Disord. 2012;140(2):113-24

12. Talarowska M, Florkowski A, Zboralski K, et al. Auditory-verbal declarative and operating memory among patients suffering from depressive disorders - preliminary study. Adv Med Sci. 2010;55(2):317-27.

13. Talarowska M, Zboralski K, Gałecki P. Correlations between working memory effectiveness and depression levels after pharmacological therapy. Psychiatr Pol. 2013;47(2):255-67.

14. Papakostas $\mathrm{Gl}$. Cognitive symptoms in patients with major depressive disorder and their implications for clinical practice. J Clin Psychiatry. 2013; 75(1):8-14.

15. Trivedi MH, Rush AJ, Wisniewski SR, et al. Evaluation of outcomes with citalopram for depression using measurement based care in STAR*D: implications for clinical practice. Am J Psychiatry. 2006;163(1): 28-40.

16. Nemeroff CB, Owens MJ. Treatment of mood disorders. Nat Neurosci. 2002; 5:1068-70.

17. Bet PM, Hugtenburg JG, Penninx BW, Hoogendijk WJ. Side effects of antidepressants during long-term use in a naturalistic setting. Eur Neuropsychopharmacol. 2013;23(11):1443-51.

18. Zhang ZJ, Kang WH, Li Q, Tan QR. The beneficial effects of the herbal medicine Free and Easy Wanderer Plus (FEWP) for mood disorders: doubleblind, placebo-controlled studies. J Psychiatr Res. 2007;41(10):828-36.

19. An L, Zhang YZ, Yu NJ, et al. The total flavonoids extracted from XiaobuxinTang up-regulate the decreased hippocampal neurogenesis and neurotrophic molecules expression in chronically stressed rats. Prog NeuroPsychopharmacol Biol Psychiatry. 2008;32(32):1484-90.

20. Hu Y, Liu P, Guo DH, Rahman K, Wang DX, Xie TT. Antidepressant effects of the extract YZ-50 from Polygala tenuifolia in chronic mild stress treated rats and its possible mechanisms. Pharm Biol. 2010;48:794-800.

21. Orsolini L, Bellantuono C. Serotonin reuptake inhibitors and breastfeeding: a systematic review. Hum Psychopharmacol. 2015;30(1):4-20.

22. Ge L, Liu L, Liu H, et al. Resveratrol abrogates lipopolysaccharide-induced depressive-like behavior, neuroinflammatory response, and CREB/BDNF signaling in mice. Eur J Pharmacol. 2015;768:49-57.

23. Pliakas AM, Carlson RR, Neve RL, et al. Altered responsiveness to cocaine and increased immobility in the forced swim test associated with elevated CAMP response element-binding protein expression in nucleus accumbens. J Neurosci. 2001;21(18):7397-403.

24. Conti AC, Cryan JF, Dalvi A, et al. CAMP response element-binding protein is essential for the upregulation of brain-derived neurotrophic factor transcription, but not the behavioral orendocrine responses to antidepressant drugs. J Neurosci. 2002;22(8):3262-8.

25. Yao H, Gu LJ, Guo JY. Study on effect of astragali radix polysaccharides in improving learning and memory functions in aged rats and its mechanism. Zhongguo Zhong Yao Za Zhi. 2014;39(11):2071-5.

26. Heine VM, Maslam S, Zareno J, Joëls M, Lucassen PJ. Suppressed proliferation and apoptotic changes in the rat dentate gyrus after acute and chronic stress are reversible. Eur J Neurosci. 2004;19(1):131-44.

27. Willner P, Towell A, Sampson D, Sophokleous S, Muscat R. Reduction of sucrose preference by chronic unpredictable mild stress, and its restoration by a tricyclic antidepressant. Psychopharmacol. 1987;93(3):358-64

28. Lepine JP, Briley M. The increasing burden of depression. Neuropsychiatr Dis Treat. $2011 ; 7(1): 3-7$.

29. de Paula JJ, Bicalho MA, Ávila RT, Cintra MT, Diniz BS, Romano-Silva MA, MalloyDiniz LF. A Reanalysis of Cognitive-Functional Performance in Older Adults: Investigating the Interaction Between Normal Aging, Mild Cognitive Impairment, Mild Alzheimer's Disease Dementia, and Depression. Front Psychol. 2016;6:2061. 
30. Butters MA, Whyte EM, Nebes RD, Begley AE, Dew MA, Mulsant BH, et al The nature and determinants of neuropsychological functioning in late-life depression. Arch Gen Psychiatry. 2004;61(6):587-95.

31. Sexton CE, McDermott L, Kalu UG, Herrmann LL, Bradley KM, Allan CL, et al. Exploring the pattern and neural correlates of neuropsychological impairment in late-life depression. Psychol Med. 2012;42(6):1195-202.

32. dePaula JJ, Miranda DM, Nicolato R, Moraes EN, Bicalho MA, Malloy-Diniz LF. Verbal learning on depressive pseudodementia: accentuate impairment of free recall, moderate on learning processes, and spared short-term and recognition memory. Arq Neuropsiquiatr. 2013;71(9A):596-9.

33. Naismith SL, Hickie IB, Turner K, Little CL, Winter V, Ward PB, et al. Neuropsychological performance in patients with depression is associated with clinical, etiological and genetic risk factors. J Clin Exp Neuropsychol. 2003;25(6):866-77.

34. Mahar I, Bambico FR, Mechawar N, Nobrega JN. Stress, serotonin, and hippocampal neurogenesis in relation to depression and antidepressant effects. Neurosci Biobehav Rev. 2013;38:173-92.

35. Yalcin I, Aksu F, Belzung C. Effects of desipramine and tramadol in a chronic mild stress model in mice are altered by yohimbine but not by pindolol. Eur J Pharmacol. 2005;514(2-3):165-74.

36. Pothion S, Bizot JC, Trovero F, Belzung C. Strain differences in sucrose 374 preference and in the consequences of unpredictable chronic mild stress. Behav Brain Res. 2004;155(1):135-46.

37. DSM-IV. Diagnostic and statistical manual of mental disorders. 4th ed. Washington D.C: American Psychiatric Association; 2000.

38. Kushwah N, Jain V, Deep S, et al. Neuroprotective Role of Intermittent Hypobaric Hypoxia in Unpredictable Chronic Mild Stress Induced Depression in Rats. Plos One. 2016;11(2):e0149309.

39. Eichenbaum $\mathrm{H}$. The hippocampus and declarative memory: cognitive mechanisms and neural codes[J]. Behav Brain Res. 2001;127(1-2):199-207.

40. Eichenbaum $\mathrm{H}$. The hippocampus and mechanisms of declarative memory [J]. Behav Brain Res. 1999;103(2):123-33.

41. Trivedi MH, Rush AJ, Wisniewski SR, et al. Evaluation of outcomes with citalopram for depression using measurement-based care in STAR*D: implications for clinical practice.[J]. Am J Psychiatr. 2006;163(1):28-40.

42. Olton DS, Paras BC. Spatial memory and hippocampal function [J]. Neuropsychologia. 1979;17(6):669-82

43. Matthews K, Forbes N, Reid IC. Sucrose consumption as a hedonic measure following chronic unpredictable mild stress. Physiol Behav. 1995;57(2):241-8.

44. Heberlein A, Lenz B, Muschler M, et al. BDNF plasma levels decrease during benzodiazepine withdrawal in patients suffering from comorbidity of depressive disorder and benzodiazepine dependence. Psychopharmacology. 2010;209(2):213-5.

45. Scearce LK. Monitoring spatial learning and memory in Alzheimer's disease mouse models using the Morris Water Maze. Methods Mol Biol. 2011;670: 191-205.

46. Bondi CO, Rodriguez G, Gould GG, Frazer A, Morilak DA. Chronic unpredictable stress induces a cognitive deficit and anxiety-like behavior in rats that is prevented by chronic antidepressant drug treatment. Neuropsychopharmacology. 2008;33(2): 320-31.

47. Hu Y, Yin WG, Lin R, Li W. Comparison of brain-derived neurotrophic factor levels in hippocampus and serum in two rat models of depression. Zhong Guo Lao Nian Xue Za Zhi. 2009;29:2188-90 (In Chinese).

48. Wei KL, Cheng YM, Sang WH, et al. Comparative study of duloxetine and paroxetine in treating depression with different symptoms. Zhongguo Lin Chuang Yao Li Xue Za Zhi. 2011;27:252-4 (In Chinese).

49. Yang M, Wen SY, Wu MC. Improvement in negative emotion and inflammatory factor levels in chronic heart failure patients after paroxetine treatment. Zhong Guo Yao Fang. 2013;24:3433-5 (In Chinese).

50. Fei $H Z$, Wang $H, H u X Y$, et al. Improvement in oxidative stress, HPA axis function, and hippocampal brain-derived neurotrophic factor expression after paroxetine treatment. Zhong Guo Lin Chuang Yao Li Xue Za Zhi. 2012; 17:1137-42 (In Chinese).

51. Skirzewski M, Hernandez L, Schechter LE, et al. Acute lecozotan administration increases learning and memory in rats without affecting anxiety or behavioral depression. Pharmacol Biochem Behav. 2010;95(3):325-30.

52. Yoshimura R, Ikenouchi Sugita A, Hori H, et al. Blood levels of brain-derived neurotrophic factor (BDNF) in major depressive disorder. Seishin Shinkeigaku Zasshi. 2010;112(10):982-5.
53. Butters MA, Young JB, Lopez O, Aizenstein HJ, Mulsant BH, Reynolds CFIII, et al. Pathways linking late-life depression to persistent cognitive impairment and dementia. Dialogues Clin Neurosci. 2008;10(3):345-57.

54. Naismith SL, Norrie LM, Mowszowski L, Hickie IB. The neurobiology of depression in later-life: clinical, neuropsychological, neuroimaging and pathophysiological features. Prog Neurobiol. 2012;98(1):99-143.

55. Panza F, Frisardi V, Capurso C, D'Introno A, Colacicco AM, Imbimbo BP, et al. Late-Life depression, mild cognitive impairment, and dementia: PossibleContinuum? Am J Geriatr Psychiatry. 2010;18(2):98-116.

56. Tham MW, Woon PS, Sum MY, Lee TS, Sim K. White matter abnormalities in major depression: evidence from post-mortem, neuroimaging and geneticstudies. J Affect Disord. 2011;132(1-2):26-36.

57. Wang C, Guo R, Zhu X. Effect of XingpiJieyu Decoction on learning-memory behavior and inflammatory factors level in depression rats. J Tradit Chin Med. 2014;7:503-6.

58. Troadec JD, Marien M, Mourlevat S, Debeir T, Ruberg M, et al. Activation of the mitogen-activated protein kinase (ERK(1/2)) signaling pathway by cyclic AMP potentiates the neuroprotective effect of the neurotransmitter noradrenaline on dopaminergic neurons. Mol Pharmacol. 2002;62(5):1043-52.

59. Meyer-Franke A, Kaplan MR, Pfrieger FW, Barres BA. Characterization of the signaling interactions that promote the survival and growth of developing retinal ganglion cells in culture. Neuron. 1995;15(4):805-19.

60. Cui Q, So KF. Involvement of CAMP in neuronal survival and axonal regeneration. Anat Sci Int. 2004;79(4):209-12.

61. Domeniconi M, Filbin MT. Overcoming inhibitors in myelin to promote axonal regeneration. J Neurol Sci. 2005;233(1-2):43-7.

62. Hannila SS, Filbin MT. The role of cyclic AMP signaling in promoting axonal regeneration after spinal cord injury. Exp Neurol. 2008;209(209):321-32.

63. Ming GL, Song HJ, Berninger B, Holt CE, Tessier-Lavigne M, et al. CAMPdependent growth cone guidance by netrin-1. Neuron. 1997;19(6):1225-35.

64. Rodger J, Goto H, Cui Q, Chen PB, Harvey AR. cAMP regulates axon outgrowth and guidance during optic nerve regeneration in goldfish. Mol Cell Neurosci. 2005:30(3):452-64.

65. Li YF, Cheng YF, Huang Y, et al. Phosphodiesterase-4D knock-out and RNA interference-mediated knock-down enhance memory and increase hippocampal neurogenesis via increased CAMP signaling. J Neurosci. 2011; 31(1):172-83.

66. Manji HK, Duman RS. Impairments of neuroplasticity and cellular resilience in severe mood disorders: implications for the development of novel therapeutics. Psychopharmacol Bull. 2001;35(2):5-49.

67. Wang C, Yang XM, Zhuo YY, et al. The phosphodiesterase-4 inhibitor rolipram reverses $A \beta$-induced cognitive impairment and neuroinflammatory and apoptotic responses in rats. Int J Neuropsychopharmacol. 2012;15(6): 749-66.

68. Soto I, Rosenthal JJ, Blagburn JM, Blanco RE. Fibroblast growth factor 2 applied to the optic nerve after axotomy up-regulates BDNF and TrkB in ganglion cells by activating the ERK and PKA signaling pathways. J Neurochem. 2006:96(1):82-96.

69. Hashimoto K, Shimizu E, lyo M. Critical role of brain-derived neurotrophic factor in mood disorders. Brain Res Brain Res Rev. 2004;45(2):104-14.

70. Malberg JE, Blendy JA. Antidepressant action: to the nucleus and beyond. Trends Pharmacol Sci. 2005;26(12):631-8.

71. Faes C, Aerts M, Geys $H$, et al. Modeling spatial learning in rats based on Morris water maze experiments. Pharm Stat. 2010:9(1):10-20.

72. Hayward P. Presenilin dysfunction leads to memory and $p$ lasticit defects. Lancet Neurol. 2004;3(6):327.

73. Shi YQ, Huang TW, Chen LM, Pan XD, Zhang J, Zhu YG, Chen XC. Ginsenoside rg1attenuates amyloid-beta content, regulates pka/creb activity, and improves cognitive performance in samp8 mice. J Alzheimers Dis Jad. 2010;19(3):977-89.

74. Ding G, Yu G, Wu Y, et al. Effects of Jiawei Xiaoyao decoction on CAMP, PKA, and PKC levels in hippocampus of depression rats. Zhong Guo Shi Yan Fang Ji Xue Za Zhi. 2012;18:162-4 (In Chinese).

75. Castren E, Rantamaki T. The role of BDNF and its receptors in depression and antidepressant drug action: reactivation of developmental plasticity. Dev Neurobiol. 2010;70:289-97

76. Bocchio-Chiavetto L, Bagnardi V, Zanardini R, Molteni R, Nielsen MG Placentino A, et al. Serum and plasma BDNF levels in major depression: a replication study and meta-analyses. World J Biol Psychiatry. 2010; $11(6): 763-73$ 
77. Chen B, Dowlatshahi D, MacQueen GM, Wang JF, Young LT. Increased hippocampal BDNF immunoreactivity in subjects treated with antidepressant medication. Biol Psychiatry. 2001;50(4):260-5.

78. Lang UE, Hellweg R, Gallinat J. BDNF serum concentrations in healthy volunteers are associated with depression-related personality traits. Neuropsychopharmacology. 2004;29(4):795-8.

79. Russo-Neustadt A, Ha T, Ramirez R, Kesslak JP. Physical activityantidepressant treatment combination: impact on brain-derived neurotrophic factor and behavior in an animal model. Behav Brain Res. 2001;120(1):87-95.

80. Rasmusson AM, Shi L, Duman R. Downregulation of BDNF mRNA in the hippocampal dentate gyrus after re-exposure to cues previously associated with footshock. Neuropsychopharmacology. 2002;27(2):133-42.

81. Itoh T, Tokumura M, Abe K. Effects of rolipram, a phosphodiesterase 4 inhibitor, in combination with imipramine on depressive behavior, CRE-binding activity and BDNF level in learned helplessness rats. Eur J Pharmacol. 2004:498(1-3):135-42

82. Pizarro JM, Lumley LA, Medina W, Robison CL, Chang WE, Alagappan A, et al. Acute social defeat reduces neurotrophin expression in brain cortical and subcortical areas in mice. Brain Res. 2004;1025(1-2):10-20.

83. Schmidt HD, Duman RS. Peripheral BDNF produces antidepressant-like effects in cellular and behavioral models. Neuropsychopharmacology. 2010;35(12):2378-91.

84. Meng C, He Z, Xing D. Low-level laser therapy rescues dendrite atrophy via upregulating bdnf expression: Implications for alzheimer's disease. J Neurosci. 2013:33(33):13505-17.

85. Tao X, Finkbeiner S, Arnold D, Shaywitz A, Greenberg M. Ca2+ influx regulates bdnf transcription by a creb family transcription factor-dependent mechanism. Neuron. 1998;20(4):709-26.

86. Reichardt LF. Neurotrophin-regulated signalling pathways. Philos Trans R Soc Lond B Biol Sci. 2006;361(1473):1545-64.

87. Yossifoff M, Kisliouk T, Meiri N. Dynamic changes in DNA methylation during thermal control establishment affect CREB binding to the brainderived neurotrophic factor promoter. Eur J Neurosci. 2008;28(11):2267-77.

88. Ye $Y$, Wang $G$, Wang $H$, et al. Brain-derived neurotrophic factor (BDNF) infusion restored astrocytic plasticity in the hippocampus of a rat model of depression. Neurosci Lett. 2011:503(1):15-9.

89. Vinet J, Carra S, Blom JM, et al. Chronic treatment with desipramine and fluoxetine modulate BDNF, CaMKK-alpha and CaMKK-beta mRNA levels in the hippocampus of transgenic mice expressing antisense RNA against the glucocorticoid receptor. Neuropharmacology. 2004;47(7):1062-9.

90. Yulug B, Ozan E, Gonl AS, Kilic E. Brain-derived neurotrophic factor, stress and depression: a mini review. Brain Res Bull. 2009;78(6):267-9.

91. Wang C, Zhang J, Lu Y, et al. Antidepressant-like effects of the phosphodiesterase-4 inhibitor etazolate and phosphodiesterase-5 inhibitor sildenafil via cyclic AMP or cyclic GMP signaling in mice. Metab Brain Dis. 2014;29(3):673-82.

92. Zhao $\mathrm{H}$, Li Q, Pei X, et al. Long-term ginsenoside administration prevents memory impairment in aged C57BL/6 J mice by up-regulating the synaptic plasticity-related proteins in hippocampus. Behav Brain Res. 2009;201(2):311-7

93. Wang C, Guo R. The Effect of Xingpijieyu Decoction on Depressive Behavior and Serum 5-HT as well as Corticosterone of Depression Rats from Chronic Stress. J Tradit Chin Med. 2014;12:1633-5.

\section{Submit your next manuscript to BioMed Central and we will help you at every step:}

- We accept pre-submission inquiries

- Our selector tool helps you to find the most relevant journal

- We provide round the clock customer support

- Convenient online submission

- Thorough peer review

- Inclusion in PubMed and all major indexing services

- Maximum visibility for your research

Submit your manuscript at www.biomedcentral.com/submit
C) Biomed Central 